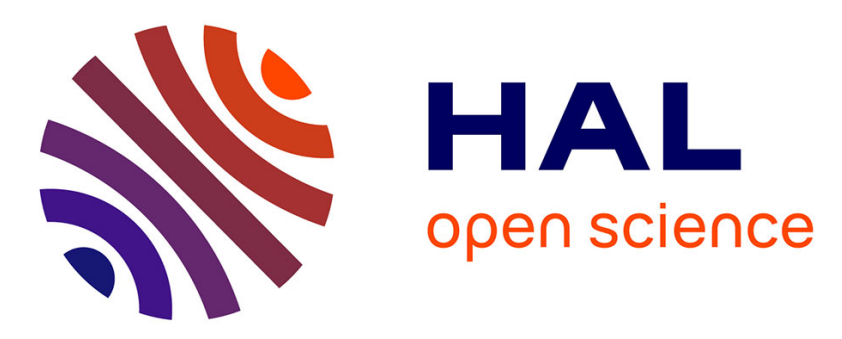

\title{
Numerical divergent series resummation in fluid flow simulation
}

\author{
Dina Razafindralandy, Aziz Hamdouni, Cyrille Allery
}

\section{To cite this version:}

Dina Razafindralandy, Aziz Hamdouni, Cyrille Allery. Numerical divergent series resummation in fluid flow simulation. Revue Européenne de Mécanique Numérique/European Journal of Computational Mechanics, 2008, 17 (4), pp.431-451. hal-00315330

\section{HAL Id: hal-00315330 \\ https://hal.science/hal-00315330}

Submitted on 1 Apr 2019

HAL is a multi-disciplinary open access archive for the deposit and dissemination of scientific research documents, whether they are published or not. The documents may come from teaching and research institutions in France or abroad, or from public or private research centers.
L'archive ouverte pluridisciplinaire HAL, est destinée au dépôt et à la diffusion de documents scientifiques de niveau recherche, publiés ou non, émanant des établissements d'enseignement et de recherche français ou étrangers, des laboratoires publics ou privés. 


\title{
Numerical divergent series resummation in fluid flow simulation
}

\author{
Dina Razafindralandy, Aziz Hamdouni, Cyrille Allery \\ LEPTAB, Université de La Rochelle
}

\begin{abstract}
The perturbation theory has proved to be an efficient tool for the numerical resolution of non-linear problems in mechanics. However, it is not suitable for singular problems, for which the series solution is divergent. We propose to use the Borel-Laplace series resummation method for the resolution of such a problem. The resulting algorithm is applied to some model problems in fluid mechanics.
\end{abstract}

Keywords: Perturbation techniques, Divergent series, Borel-Laplace resummation, Asymptotic Numerical Method.

\section{Résumé}

La théorie de perturbation est un outil efficace pour la résolution numérique de problèmes non-linéaires en mécanique. Toutefois, elle n'est pas adaptée à des problèmes singuliers, pour lesquels la solution sous forme de série formelle est divergente. Nous proposons d'utiliser numériquement la méthode de resommation de séries de Borel-Laplace pour la résolution d'un tel problème. L'algorithme résultant est appliqué à la résolution de problèmes modèles en mécanique des fluides.

Mots-clés : Techniques de perturbation, Séries divergentes, Resommation de Borel-Laplace, Méthode Asymptotique Numérique.

\section{Introduction}

Based on the perturbation theory [5] and a finite element discretization, the Asymptotic Numerical Method (ANM) permits to solve many non-linear problems in structure mechanics $[11,13,12]$, and in low Reynolds number fluid flow simulation $[29,8,3]$. The efficiency of ANM, compared to Runge-Kutta-like methods, is due to its low computational cost. Indeed, the series decomposition of the solution leads to a cascade of linear problems having the same operator. 
So, only one matrix has to be inverted. Moreover, a continuation procedure can be used when the radius of convergence of the series is reached [9]. One other advantage of ANM is its ability in path following and the detection of bifurcations [3].

ANM cannot, however, be directly applied to problems having a singularity at the origin. Indeed, in this case, the radius of convergence vanishes and the continuation procedure cannot be performed. Usual extrapolation methods such as Padé approximants are also inefficient. Though, a divergent series is not uninteresting. They often appeared in astronomy problems and was used for calculations. They may also occur in fluid mechanics. Consider, for instance, the linear heat equation:

$$
\frac{\partial T}{\partial t}=\frac{\partial^{2} T}{\partial x^{2}}
$$

where $T$ is the temperature, and $t$ and $x$ are the time and space variables. If the initial condition is

$$
T(0, x)=\frac{1}{1-x}
$$

then the formal series solution, according to $t$, is [21]

$$
T(t, x)=\sum_{k \geq 0} \frac{(2 k) !}{k !} \frac{1}{(1-x)^{2 k+1}} t^{k},
$$

which is divergent at any point $x$ of the domain.

Another example is the discretized unsteady Navier-Stokes equations, which govern the motion of fluids. For these equations, a formal time series decomposition leads to an interesting cascade of linear problem where the operator does not change even if a continuation procedure is performed (see appendix). Only one matrix inversion is then necessary during the whole simulation. One the other hand, as shown in appendix, the radius of convergence decreases with the square of the number of grid points. The series is "practically" divergent, especially for turbulent flows where the number of grid points is generally very high.

The most promising tool to treat divergent series is the Borel-Laplace resummation method. This technique is a theoretical procedure developped initially to find a sectorial analytic solution from a divergent series which is a formal solution of an equation $[14,21,7]$. And if the formal series solution has a nonzero convergence radius, it can be seen as an extrapolation procedure. The method is based on the usual Laplace transformation and its inverse, the Borel transformation. Its limit is that the series must be a Gevrey series, i.e. must not "diverge faster than the factorial series". Fortunately, the majority of formal series arising from a physical problem are a Gevrey series, as proved by Maillet's theorem [22, 28].

In this article, we propose to adapt the Borel-Laplace resummation method for a numerical use in real mechanics problems, particularly in fluid mechanics. 
An algorithm will be built. However, in this pioneer work, the method will only be applied to some reduced model problems. Simplifying hypotheses are also made. The aims of this exploration phase are to check if the Borel-Laplace resummation technique is suitable for a numerical use, to identify the eventual problems and to know what can be hoped when solving a non-reduced problem. The paper will be structured as follows. In Section 2, the Borel-Laplace resummation will be very briefly exposed. In Section 3, the transposition of the theory into a numerical algorithm will be presented. Some numerical tests are carried out in Section 4. In appendix, an algorithm for the resolution of the discretized Navier-Stokes equations, based on a time series decomposition is developped and the radius of convergence is evaluated.

\section{The Borel-Laplace resummation method}

A complete presentation of the Borel-Laplace resummation theory would require the introduction of complex mathematical concepts. However, the mechanism is easy to understand and, at our stage, we do not use all these concepts. So, they will be skipped and we will illustrate the method through two examples. For complementary information, the reader can refer to the papers of Borel [6] and Gevrey [16] and to more recent papers on divergent series [5, 24, ?, 15].

\subsection{Two examples}

To understand the mechanism of the Borel-Laplace resummation method, it is sufficient to remark that, formally,

$$
\breve{s}=\sum_{k \geq 0} s_{k} z^{k}=s_{0}+\int_{0}^{+\infty}\left(\sum_{k \geq 0} \frac{s_{k+1}}{k !} \xi^{k}\right) e^{-\xi / z} \mathrm{~d} \xi
$$

for any formal series $\breve{s}=\sum_{k \geq 0} s_{k} z^{k}$, since

$$
k !=\int_{0}^{+\infty} \xi^{k} e^{-\xi} \mathrm{d} \xi
$$

If the formal series $\sum_{k \geq 0} \frac{s_{k+1}}{k !}$, called the "Borel transform" of the series $\breve{s}-s_{0}$, can be replaced by an analytic function at the origin and in a sector containing the positive real axis such that the integral in [4] exists, we obtain an analytic function in the right-hand side. This analytical function is asymptotic (in a sense which will be more precisely defined later) to the initial series at the origin.

Consider the Euler equation:

$$
z^{2} \frac{\mathrm{d} w}{\mathrm{~d} z}+w=z .
$$


The formal series solution of this equation is:

$$
\breve{w}=\sum_{k \geq 0}(-1)^{k} k ! z^{k+1} .
$$

This series is divergent. A non-optimized perturbation-method-based algorithm such as ANM would not give any analytical solution.

The Borel transform of the series [7] is

$$
\sum_{k \geq 0} \frac{w_{k+1}}{k !} \xi^{k}=\sum_{k \geq 0}(-1)^{k} \xi^{k} .
$$

This series can be extended to the analytic function $P: \xi \in \mathbb{C}-\{-1\} \mapsto \frac{1}{1+\xi}$. Hence, the right-hand side of [4], applied to $\breve{w}$, gives:

$$
1+\int_{0}^{+\infty} \frac{1}{1+\xi} e^{-\xi / z} \mathrm{~d} \xi
$$

This is an analytic function in the complex half-plane where the real part of $z$ is positive. This function is the "sum" of the divergent series [7]. It is a solution of [6] and can be computed numerically.

In summary, from the divergent series [7], the resummation could provide an analytic function which is a solution of the Euler equation.

In the following example, we consider a problem whose series solution is convergent. We then show that, in this case, the resummation method can be understood as a prolongation method.

Consider the initial value problem:

$$
\left\{\begin{array}{l}
\frac{\mathrm{d} w}{\mathrm{~d} z}+w^{2}=0 \\
w(0)=1 .
\end{array}\right.
$$

The formal series solution is:

$$
\breve{w}=\sum_{k \geq 0}(-1)^{k} z^{k} .
$$

which is convergent inside the unity disc. The Borel transform of [10] is

$$
\sum_{k \geq 0} \frac{(-1)^{k+1}}{k !} \xi^{k}
$$

We recognize that it is the Taylor expansion of the function

$$
\xi \mapsto-e^{-\xi} .
$$


With this function, the right-hand side of equation [4] is

$$
1+\int_{0}^{+\infty} e^{-\xi} e^{-\xi / z} \mathrm{~d} \xi=\frac{1}{1+z}
$$

This is the analytic solution of problem $[9]$ in $\mathbb{C}-\{-1\}$. This function prolongs the series [10].

In the following subsection, the resummation procedure in the general case is summarized.

\subsection{Summary of the resummation procedure}

Assume that the perturbation theory gives a formal series solution

$$
\breve{s}=\sum_{k \geq 1} s_{k} z^{k} \in \mathbb{C}[[z]]
$$

of a problem, where $C[[z]]$ is the space of the complex formal series in $z$. The resummation procedure contains three steps:

1. the first operation is the formal Borel transformation:

$$
\breve{s}=\sum_{k \geq 1} s_{k} z^{k} \in \mathbb{C}[[z]] \quad \longmapsto \quad \mathcal{B}(\breve{s})=\sum_{k \geq 0} \frac{s_{k+1}}{k !} \xi^{k} \in \mathbb{C}[[\xi]] ;
$$

the Borel transform $\mathcal{B}(\breve{s})$ has a non-zero convergence radius if $\breve{s}$ is a Gevrey series ${ }^{1}$, i.e. if there exists two constants $C$ and $A$ such that

$$
\forall k \geq 1, \quad\left|s_{k}\right|<C A^{k} k ! ;
$$

2. assume that $\breve{s}$ is a Gevrey series, the second operation is an extension of $\mathcal{B}(\breve{s})$, if possible, into an analytic function

$$
P: \xi \in S(a) \mapsto P(\xi) \in \mathbb{C}
$$

in a (open) sector $S(a)$ containing a half-line $d_{a}$ linking the origin to the infinity in the direction $a$;

3. the last operation is the Laplace transform in the direction $d_{a}$ which permits to go back to the original space:

$$
P \in H_{a} \mapsto \mathcal{L}_{a} P(z)=\int_{d_{a}} P(\xi) e^{-\xi / z} \mathrm{~d} \xi
$$

where $H_{a}$ is the space of functions which are analytic in a vicinity of 0 and which can be extended into an analytic function having an exponential growth in a sector $S_{a}$ containing the direction $d_{a}$, i.e.

$$
\exists c \in \mathbb{R}, \quad|P(\xi)| \leq O\left(e^{c \xi}\right) \quad \forall \xi \in S_{a} .
$$

1. Only the order 1 is considered in this article. 
In the previouos examples, $a=0$ and $d_{0}$ is the positive real half-axis.

The key idea of the Borel-Laplace resummation method is that it is easier to extend a convergent series (here, the Borel transform) outside his convergence domain than to extend a divergent series (the original series) in a non-empty domain.

To sum up, a formal series $\breve{s}=\sum_{k \geq 0} s_{k} z^{k}$ is Borel-summable in a direction $a$ if $\mathcal{B}\left(\breve{s}-s_{0}\right) \in H_{a}$. Its Borel sum is the function

$$
s_{0}+\mathcal{L}_{a}\left[\mathcal{B}\left(\breve{s}-s_{0}\right)\right] .
$$

The Borel sum [19] is analytic in the Borel disc whose one diameter is $\left[0, \frac{1}{c} e^{i a}\right]$ (see Figure 1). It is asymptotic to the original series in the following sens: a formal series $\breve{s}=\sum_{i \geq 0} s_{k} z^{k}$ is the Gevrey asymptotic expansion of a function $s$ in a sector $S$ (at the origin) if, for all compact sub-sector $T$ of $S$, there esists two real constants $C$ and $A$ such that

$$
\left|s(z)-\sum_{j=0}^{k-1} s_{j} z^{j}\right|<C A^{k}(k !)|z|^{k},
$$

for all $k \in \mathbb{N}$ and all $z \in T$.

Figure 1: Borel disc in the direction $a$

The Gevrey expansion is a generalization of the Taylor expansion for divergent series. For complementary results on the existence and uniqueness of the Gevrey expansion one can refer to Bender et al. [5], Malgrange et al. [23], Loday-Richaud [19], Costin [10] and Fruchard et al. [15].

In the following section, an algorithm for the effective numerical computation of the Borel sum is presented. 


\section{Numerical algorithm}

Consider the following problem:

$$
E(s(z), z)=0
$$

which has a formal series solution $\breve{s}, E$ being a differential operator. A numerical perturbation method such as ANM permits, by identification, to find the $l$ first terms of the series $\breve{s}$ and leads to a truncated series:

$$
\breve{s}^{l}(z)=\sum_{k=0}^{l} s_{k} z^{k}
$$

which is an approximation of the solution around $z=0$. The order $l$ of [21], on which depends the accuracy of the method, is arbitrary.

The three operations of the resummation methods are translated into a numerical algorithm as follows.

1. The first operation, the Borel transformation, is a purely algebraic operation and introduces no particular problem. It gives the following Borel transform of $\left(\breve{s}^{l}-s_{0}\right)$ :

$$
\mathcal{B}\left(\breve{s}^{l}-s_{0}\right)(\xi)=\sum_{k=0}^{l-1} \frac{s_{k+1}}{k !} \xi^{k} .
$$

2. The second operation consists in extending [22] into a function which is analytic in a direction to the infinity. It is assumed that this extension is possible. This can be performed using the usual Padé approximant procedure [5, 27]. It gives a rational approximation of [22]:

$$
P(\xi)=\frac{A_{0}+A_{1} \xi+\cdots+A_{n_{1}} \xi^{n_{1}}}{1+B_{1} \xi+\cdots+B_{n_{2}} \xi^{n_{2}}}
$$

where $n_{1}+n_{2}=l$ and $A_{i}$ and $B_{i}$ are the real numbers such that the Taylor expansion of $P$ at the order $l$ is the truncated series [22]. In opposition to the original series [21], the Padé approximant procedure is efficient on $[22]$ because this series is convergent.

3. The last operation is the application the Laplace transform $\mathcal{L}_{a} P$ of [23] in a direction where $P$ has no pole. One property of the Padé approximants [23] is that it has an exponential growth at the infinity, and consequently, the Laplace transform exists. To calculate the value of the Borel sum

$$
B L_{a}\left(\breve{s}^{l}\right)=s_{0}+\mathcal{L}_{a} P
$$

at any point $z$, a Gauss-Laguerre quadrature method $[17,18]$ is used.

Table 1 summarizes the main operations of the resummation procedure. This algorithm was integrated into a perturbation-method-based Fortran code and tested in the next section. 
Table 1: The numerical algorithm

$$
\begin{array}{cc}
\breve{s}^{l}=\sum_{k=0}^{l} s_{k} z^{k} & \operatorname{BL}_{a}\left(\breve{s}^{l}\right)(z)=s_{0}+\int_{d_{a}} P(\xi) e^{-\xi / z} \mathrm{~d} \xi \\
\text { Borel } & \uparrow \text { Laplace }\left(\mathcal{L}_{a}\right) \\
\mathcal{B}\left(\breve{s}^{l}-s_{0}\right)=\sum_{k=0}^{l-1} \frac{s_{k+1}}{k !} \xi^{k} \stackrel{\text { Padé }}{\longrightarrow} & P(\xi)=\frac{A_{0}+A_{1} \xi+\cdots+A_{N_{1}} \xi^{N_{1}}}{1+B_{1} \xi+\cdots+B_{N_{2}} \xi^{N_{2}}}
\end{array}
$$

\section{Numerical tests}

Our long-term aim is the resolution of the Navier-Stokes equations. However, in this exploration phase, we only consider some model problems. The first example is Equation [9]. The exact solution is

$$
\frac{1}{1+z} \text {. }
$$

Equation [9] is not an example on which the maximum profit of the resummation can be made because it does not present an irregularity at the origin $z=0$ but it has a quadratic non-linearity like the Navier-Stokes equations.

\subsection{Tests on Equation [9]}

In a first test, the solution given by the perturbation method alone is compared to the solution given by the association of the perturbation method and the resummation method. The series solution is computed up to the 8-th order $(l=8)$. The direction of the Laplace transformation is the real positive axis and six Gauss points are used for the Gauss-Laguerre integration.

The result of this first test is presented on Figure 2. It can be observed on it that the series solution provided by the perturbation method is no longer a good approximation from about $z=0.7$. When the perturbation method is combined to the resummation, the computed solution remains very close to the exact solution at least until $z=2.5$.

The first conclusion is that the above presented algorithm is operational. Note that no optimization operation such as convergence acceleration nor an optimization of the quadrature was performed in the algorithm. The second conclusion is that, as expected, the resummation method brings a very significant improvement to the perturbation method. In this case, the range of validity is multiplied by more than 3.5 . 
Figure 2: Solutions with and without resummation

Theoretically, the resummation method should lead to the exact solution until the infinity (Subsection 2.1) but since the initial series was truncated and no optimization was done, numerical effects become non-negligible for large values of $z$.

The next test is done on the same problem [9] but this time, a continuation procedure is introduced in order to reach high values of $z$.

Let Res be the residue:

$$
\operatorname{Res}(z)=\frac{\mathrm{d} w}{\mathrm{~d} z}(z)+w^{2}(z) .
$$

For the comparison, two calculations are done. In the first one, the perturbation method is combined to the continuation procedure (PERT+CONT) and in the second, the resummation procedure is added (PERT + RESUM + CONT). More precisely, the algorithm with resummation can be described as follows.

a. (PERT) The truncated series

$$
\breve{w}^{l}=\sum_{k=0}^{l} w_{k} z^{k}
$$

is computed using the perturbation method with $w_{0}=w(0)=1$. This provides an analytical approximation of the exact solution as long as the following relation holds:

$$
\frac{\|\operatorname{Res}(z)\|}{\left\|\breve{w}^{l}(z)\right\|}<\varepsilon
$$

where $\varepsilon$ is a small parameter and $\|\cdot\|$ is the usual Euclidian norm. Let $\bar{z}$ be the last computed values of $z$ for which the above relation holds. 
b. (RESUM) Next, the Borel sum $B L_{0}\left(\breve{w}^{l}\right)$ is computed. This provides an analytical approximation of the exact solution for $z \in\left[\bar{z}, z_{0}\right]$ where $z_{0}$ is the last value of $z$ for which the following relation holds:

$$
\frac{\|\operatorname{Res}(z)\|}{\left\|B L_{0}\left(\breve{w}^{l}\right)(z)\right\|}<\varepsilon .
$$

c. (CONT) When $z_{0}$ is reached, a continuation procedure is performed, that is, we run the algorithm again with, in step $\boldsymbol{a} ., w_{0}=w\left(z_{0}\right)$.

In PERT + CONT algorithm, step $\boldsymbol{b}$. is jumped and $z_{0}=\bar{z}$. The computation is carried out up to $z=10$, with $l=8$ and $\varepsilon=10^{-2}$.

(a)

(b)

Figure 3: Approximated solutions with continuation; a) without resummation $($ PERT + CONT $)$, b) with resummation $($ PERT + RESUM + CONT $)$

Figure 3 shows the approximate solutions. Visually, the exact solution cannot be distinguished from the computed solutions and is not presented. Left is the approximate solution without the resummation procedure. The bold points $(\bullet)$ represent the points where a continuation procedure is needed (the abscissa of these points correspond to the $z_{0}$ 's). Right is the approximate solution with the resummation procedure. The dashes correspond to the part of the solution obtained in phase $\boldsymbol{b}$. of the algorithm. It can be observed that the resummation procedure prolongs well the solution provided by the perturbation method such that much less continuation steps are needed. Indeed, only two continuation points are required to reach $z=10$ when the resummation method is applied, against eight when it is not.

Such a result should still have much more interest in the resolution of high order problems with ANM where the computation of the terms of the formal series (which needs an inversion of a matrix) has an important cost compared to the resummation procedure.

The above results corresponds to a truncation of the series at $l=8$. Other calculations was done with $l=15$. Table 2 compares the number of continuation 
points with $l=8$ and $l=15$. A very significant diminution of the number of continuation points can be observed when the resummation procedure is used.

Table 2: Number of continuation points for Equation [9]

\begin{tabular}{lcc}
\hline & $l=8$ & $l=15$ \\
\hline & & \\
PERT + CONT & 6 points & 5 points \\
PERT + RESUM + CONT & 2 points & 1 points \\
\hline
\end{tabular}

These tests on Equation [9] permitted to check that the proposed algorithm is functional and brings a good improvement to the perturbation method. In the following tests, we consider a reduced model of the Navier-Stokes equations for the simulation of realistic fluid flows.

\subsection{Reduced model of the Navier-Stokes equations}

The Navier-Stokes equations (see appendix, Equation [29]) are reduced using the Proper Orthogonal Decomposition (POD) method which consists in decomposing the velocity field $u$ in a particular basis $\left(\Phi^{i}\right)_{i=1, \ldots, \infty}$, composed of divergent free functions, and which maximizes the energy (see Lumley [20], Sirovich [26], Aubry [4]):

$$
u(t, x)=\sum_{i=1}^{+\infty} \varrho^{i}(t) \Phi^{i}(x)
$$

$t$ and $x$ are respectively the time and space variables. The components $\varrho^{i}$ of $u$ will be called the modes. The basis functions are generally determined numerically or experimentally and depend on the configuration of the flow.

In practice, the decomposition [26] is truncated at an order $m$. This leads to the following reduced model of the Navier-Stokes equations:

$$
\frac{\mathrm{d} \varrho^{i}}{\mathrm{~d} t}+\sum_{j, l=1}^{m} Q_{j l}^{i} \varrho^{j} \varrho^{l}+\sum_{j=1}^{m} L_{j}^{i} \varrho^{j}=F^{i}(t), \quad i=1,2, \ldots m .
$$

where $Q, L$ and $F$ are known tensors depending on the basis functions. A property of POD is that very few modes are needed to capture almost the whole energy of the flow.

In our case, the basis functions were computed numerically. They are not presented here but can be found in [1].

Equations [27] are solved using the perturbation-resummation algorithm $($ PERT + RESUM + CONT $)$ described above. For comparison, another calculation will be done using the usual adaptive 5-order Runge-Kutta method. The RungeKutta step is chosen such that the estimated value $E$ of the truncation error 
Figure 4: Geometry of the driven cavity

verifies the following relation:

$$
\frac{E}{\|\varrho(t)\|} \leq \varepsilon
$$

where $\varrho=\left(\varrho^{i}\right)_{i}$ and $\varepsilon$ is the same error parameter used in [24] and [25]. Notice that the Runge-Kutta method is of order 5 but the order of the perturbationresummation method, which is defined by $l$, can be chosen arbitrarily. Moreover, while the Runge-Kutta method gives a point by point solution, the perturbationresummation method provides an analytic solution.

The first test is carried out on an air flow inside a two-dimensional driven cavity, represented on Figure 4 . The lid velocity $U_{r e f}$ is horizontal, with $U_{r e f}=$ $(0.15 \mathrm{~m} / \mathrm{s}, 0 \mathrm{~m} / \mathrm{s})$.

$l$ is set to 10. The Laplace direction is the positive real axis (it is then assumed that the Padé approximants have no pole on this axis). Four or ten modes $(m=4$ or 10$)$ are computed. The first two modes for $t$ from 0 to 20 seconds are presented on Figure 5. A projection on the basis $\left(\Phi^{i}\right)_{i}$ of a numerical solution of the Navier-Stokes equations is used as a reference solution.

It can be observed on Figure 5 that the algorithm PERT + CONT + RESUM gives approximately the same numerical results as the Runge-Kutta method at the points where a Runge-Kutta solution is available. The difference with the reference solution may be essentially due to the numerical computation of the reference solution but the POD modelisation may also introduce some errors (truncation of the series...). Stabilization procedures exist (but not used here) to attenuate the POD modeling errors. The difference with the reference solution is then independent of the performance of our two algorithms.

Table 3 compares the number of steps needed to reach 20,50 and 100 seconds for $m=4$ modes $(l=10)$. It shows that the perturbation-resummation method 
PSfrag replacements

(a)

(b)

$\varrho^{t} \varrho^{1}$

Figure 5: Computed values of $\varrho^{1}$ (a) and $\varrho^{2}$ (b) versus $t$

Reference solution, • • Runge-Kutta, - . - Perturbationresummation

requires much less steps than the Runge-Kutta method, about 35\% less to reach 100 seconds.

Table 3: Number of continuation steps for the driven cavity, with $m=4$

\begin{tabular}{lccc}
\hline & 20 seconds & 50 seconds & 100 seconds \\
\hline & 18 steps & 41 steps & 80 steps \\
Runge-Kutta & 9 steps & 24 steps & 52 steps \\
\hline
\end{tabular}

The gain of the perturbation-resummation method increases with the number of modes. Indeed, as observed on Table 4 with $m=10$ modes, the perturbation-resummation method requires about $42 \%$ less continuation steps than the Runge-Kutta method to reach 100 seconds.

Table 4: Number of continuation steps for the driven cavity, with $m=10$

\begin{tabular}{lccc}
\hline & 20 seconds & 50 seconds & 100 seconds \\
\hline Runge-Kutta & & & \\
Perturbation-resummation & 29 steps & 81 steps & 175 steps \\
& 16 steps & 44 steps & 102 steps \\
\hline
\end{tabular}


Figure 6: Geometry of the ventilated room

Since the reconstituted flows obtained from the perturbation-resummation method and from the Runge-Kutta method are qualitatively the same, they are not presented in this article but can be found in [1].

From the above results, it can be concluded that the perturbation-resummation method provides an approximate solution as precise as the one given by the Runge-Kutta method, but with less steps. In addition, the approximate solution is analytic. These results also show that the perturbation-resummation method can be used for the simulation of realistic problems. Finally, it can be expected that the advantage of the method will be more important while simulating a high order problem such as the discretized Navier-Stokes equations.

The last test is carried out on the flow inside the $2 \mathrm{D}$ ventilated room presented on Figure 6. This geometry is used in [2] for the study of pollution in building field. For this geometry, the inlet and outlet heights are $0.31 \mathrm{~m}$. The inlet is at $0.07 \mathrm{~m}$ from the ceiling and the outlet at $0.07 \mathrm{~m}$ above the floor. The inlet velocity is $0.443 \mathrm{~m} / \mathrm{s}$.

A first simulation is done until the flow is stable. Then, a particle cloud is injected. The details of the simulation can be found in [2] and will not be reproduced here. Our interest is the comparison of the perturbation-resummation method with the Runge-Kutta method.

The flow is solved with POD, with $m=4$ modes. Table 5 presents the number of steps needed by the two methods 20,30 and 100 seconds after the injection of the particles. Also for this flow, the perturbation-resummation method requires less steps than the Runge-Kutta method, about $44 \%$ less to reach 100 seconds. The reconstituted flow can be found in [2, 25].

\section{Conclusion}

It was shown that the Borel-Laplace resummation method can be used in a numerical algorithm. Applied to a theoretical problem, it has proved to bring a 
Table 5: Number of continuation steps for the ventilated room

\begin{tabular}{lccc}
\hline & 20 seconds & 30 seconds & 100 seconds \\
\hline & & & \\
Runge-Kutta & 93 steps & 137 steps & 397 steps \\
Perturbation-resummation & 55 steps & 78 steps & 224 steps \\
\hline
\end{tabular}

really interesting improvement as a prolongation technique to the perturbation method. A prolongation may considerably reduce the computational cost when simulating a high dimensional problem such as the discretized Navier-Stokes equations where the computation of the terms of the series requires an important computation time. It was also seen from tests on reduced but realistic problems that the combination of the perturbation method to the resummation technique presents interesting advantages compared to the usual Runge-Kutta method. The perturbation-resummation technique requires less continuation steps. So, it can be hoped that, once used on high dimensional problems, it will require less matrix inversions.

In this article, no comparison on the CPU has been presented. The reason is that the Runge-Kutta method is known for a long time and has been well optimized. It is not the case of the resummation technique which was used numerically for the first time. Optimizations could be brought for example in the computation of the (vector) Padé approximants or the Laplace transform (the direction of integration, the effective calculation of the integral, ...). In addition, all the operations in the resummation technique are formal. It may then be profitable to use a symbolic software such as Maple for the resummation step instead of a purely numeric program. This should significantly reduce the computation errors.

\section{References}

[1] C. Allery. Contribution à l'identification des bifurcations et à l'étude des écoulements fluides par des systèmes dynamiques d'ordre faible (P.O.D.). $\mathrm{PhD}$ thesis, 2002.

[2] C. Allery, C. Béghein, and A. Hamdouni. Applying Proper Orthogonal Decomposition to the computation of particle dispersion in a two-dimensional ventilated cavity. Communications in Nonlinear Science and Numerical Simulation, 10:907-920, 2005.

[3] C. Allery, J-M. Cadou, A. Hamdouni, and D. Razafindralandy. Application of the asymptotic numerical method to the Coanda effect study. European Journal of Computational Mechanics, 13(1-2):57-77, 2004.

[4] N. Aubry, P. Holmes, J. Lumley, and E. Stone. The dynamics of coherent structures in the wall region of a turbulent boundary layer. Journal of Fluid Mechanics, 192:115-173, 1988. 
[5] C. Bender and S. Orszag. Advanced mathematical methods for scientists and engineers : Asymptotic methods and perturbation theory. McGraw-Hill, 1978.

[6] E. Borel. Mémoire sur les séries divergentes. Annales scientifiques de l'E.N.S. 3ème série, 16:9-131, 1899.

[7] D.J. Broadhurst and D. Kreimer. Combinatoric explosion of renormalization tamed by hopf algebra: 30-loop padé-borel resummation. Physics Letters B, 475 (1-2):63-70, 2000.

[8] J-M. Cadou. Méthode asymptotique numérique pour le calcul des branches solutions et des instabilités dans les fluides et pour les problèmes d'intéraction fluide-structure. $\mathrm{PhD}$ thesis, 1997.

[9] B. Cochelin. A path-following technique via an asymptotic-numerical method. Computers And Structures, 53(5):1181-1192, 1994.

[10] O. Costin. On Borel summation and Stokes phenomena for rank one nonlinear systems of ODE's. Duke Mathematical Journal, 93(2):289-344, 1998.

[11] N. Damil and M. Potier-Ferry. A new method to compute perturbed bifurcations : application to the buckling of imperfect elastic structures. International Journal of Engineering Science, 28(9):943-957, 1990.

[12] E-M. Daya and M. Potier-Ferry. A numerical method for non-linear eigenvalue problems. Application to vibrations of viscoelastic structures. Computers And Structures, 79:533-541, 2001.

[13] A. Elhage-Hussein, M. Potier-Ferry, and N. Damil. A numerical continuation method based on Padé approximants. International Journal of Solids ans Structures, 37:6981-7001, 2000.

[14] J. Ellis, E. Gardi, M. Karliner, and M. Samuel. Padé approximants, Borel transforms and renormalons: the Bjorken sum rule as a case study. Physics Letters B, 366(1-4):268-275, 1996.

[15] A. Fruchard and C. Zang. Remarques sur les développement asymptotiques. Annales de la Faculté des Sciences de Toulouse, 8(1):91-114, 1999.

[16] M. Gevrey. Sur la nature analytique des solutions des équations aux dérivées partielles. Premier mémoire. Annales scientifiques de l'E.N.S. $3^{e ̀ m e}$ série, 35:129-190, 1918.

[17] C. Guilpin. Manuel de calcul numérique appliqué. EDP Sciences, 1999.

[18] M. Kzaz. Convergence acceleration of the Gauss-Laguerre quadrature formula. Applied Numerical Mathematics, 29:201-220, 1999.

[19] M. Loday-Richaud. Séries formelles provenant de systèmes différentiels linéaires méromorphes. In Centre de mathématiques, editor, Journées $X$ UPS 1991, pages 69-100, 1991.

[20] J. Lumley. The structure of inhomogeneous turbulent flows. In A. Yaglom and V. Tatarski, editors, Atmospheric Turbulence and Radio Wave Propagation, pages 166-178, 1967. 
[21] D.A. Lutz, M. Miyake, and R. Schäfke. On the Borel summability of divergent solutions of the heat equation. Nagoya Mathematical Journal, 154:129, 1999 .

[22] E. Maillet. Sur les séries divergentes et les équations différentielles. Annales scientifiques de l'E.N.S. 3ème série, 20:487-518, 1903.

[23] B. Malgrange and J-P Ramis. Fonctions multisommables. Annales de l'Institut Fourier, 42(1-2):353-368, 1992.

[24] J-P. Ramis. Séries divergentes et théories asymptotiques. In Journées XUPS 1991, pages 7-67, 1991.

[25] D. Razafindralandy. Contribution à l'étude mathématique et numérique de la simulation des grandes échelles. Phd thesis, Université de La Rochelle, 2005.

[26] L. Sirovich. Turbulence and the dynamics of coherent structures, Part I: Coherent strucures, Part II : Symmetries and transformations, Part III : Dynamics and scaling. Quarterly of Applied Machanics, 45(3):561-590, 1987.

[27] S.P. Suetin. Padé approximants and efficient analytic continuation of a power series. Russian Mathematical Surveys, 57(1):43-141, 2002.

[28] J. Thomann. Formal and numerical summation of formal power series solutions of ODE's. Technical report, CIRM Luminy, 2000.

[29] A. Tri, B. Cochelin, and M. Potier-Ferry. Résolution des équations de Navier-Stokes et détection des bifurcations stationnaires par une méthode asymptotique numérique. European Journal of Computational Mechanics, 5(4), 1996.

\section{A Time series expansion for the Navier-Stokes equations}

In this appendix, an ANM algorithm obtained from a time series expansion for the discretized Navier-Stokes equations is presented. Next, a study on the convergence radius of the series is carried out.

\section{A.1 Presentation of the algorithm}

Consider a Newtonial incompressible fluid with, for simplicity, a density 1. The motion of this fluid is governed by the Navier-Stokes equations (in a dimensionless form):

$$
\left\{\begin{array}{l}
\frac{\partial u}{\partial t}+\operatorname{div}(u \otimes u)+\nabla p-\frac{1}{R e} \nabla^{2} u=0 \\
\operatorname{div} u=0
\end{array}\right.
$$


with the initial condition

$$
u(t=0, x)=u_{0}(x)
$$

where $t$ is the time variable, $x$ the space variable, $u$ the velocity field, $p$ the pressure and $R e$ the Reynolds number. After a suitable discretization, these equations are transformed into a matrix equation:

$$
M \frac{\partial U}{\partial t}+Q(U, U)+L U=0
$$

with

$$
U(t=0)=U_{0}
$$

where $U$ (respectively $U_{0}$ ) are the vectors containing the nodal values of $u$ and $p$ (resp. $\left.u_{0}\right), M$ the mass matrix, $L=\left(L_{j}^{i}\right)_{i, j=1, \ldots, N}, Q$ a vectorial quadratic operator such that

$$
Q_{i}(V, U)=\sum_{j=1}^{N} \sum_{l=1}^{N} \mathbb{Q}_{j l}^{i} V^{j} U^{l},
$$

$\mathbb{Q}=\left(\mathbb{Q}_{j, l}^{i}\right)_{i, j, l=1, \ldots, N}$ being a three-order tensor and $N$ is the number of grid points.

The unknown vector $U$ is decomposed into a formal time series as follows:

$$
U(t)=U_{0}+U_{1} t+U_{2} t^{2}+\cdots+U_{k} t^{k}+\cdots
$$

Injecting the decomposition [32] in the matrix equation [30] and identifying according to the powers of $t$, one obtains the following cascade of linear equations:

$$
\left\{\begin{array}{cc}
\text { Order } 0: & M U_{1}+Q\left(U_{0}, U_{0}\right)+L U_{0}=0 \\
\text { Order 1: } & 2 M U_{2}+Q\left(U_{0}, U_{1}\right)+Q\left(U_{1}, U_{0}\right)+L U_{1}=0 \\
\vdots & (k+1) M U_{k+1}+\sum_{r=0}^{k} Q\left(U_{r}, U_{k-r}\right)+L U_{k}=0 \\
\text { Order } k: & \vdots
\end{array}\right.
$$

This cascade of problems has the property that the equations have the same matrix to be inverted, namely $M$. The resolution of these equations provides an approximate solution in the domain of validity of the series [32]. But compared to other algorithms of the ANM family, the time-series decomposition presents one more very interesting advantage: the continuation procedure does not require the inversion of any other matrix because $M$ does not depend on the continuation points. $M$ is then inverted only once during the computation; that 
presents an important save of computational time. Only the second members change.

The performance of an algorithm built from this time series decomposition lies however on the convergence radius of the series [32]. In what follows, a analysis of the convergence radius is done.

\section{A.2 Estimation of the radius of convergence}

For simplification, Equation [30] is rewritten in the following form:

$$
\frac{\mathrm{d} U}{\mathrm{~d} t}+Q(U, U)+L U=0 .
$$

In a vectorial form, the unknown is

$$
U=\left(U^{1}, U^{2}, \ldots, U^{N}\right)^{T} .
$$

The time series decomposition [32] gives, for each component $U^{i}, i=1, \ldots, N$ :

$$
U^{i}(t)=U_{0}^{i}+U_{1}^{i} t+U_{2}^{i} t^{2}+\cdots+U_{k}^{i} t^{k}+\cdots .
$$

The initial condition is

$$
U_{0}=\left(U_{0}^{1}, U_{0}^{2}, \ldots, U_{0}^{N}\right)^{T} .
$$

We denote

$$
\begin{array}{ll}
\mathbf{Q} & =\max _{i, j, l=1, \ldots, N}\left|\mathbb{Q}_{j l}^{i}\right|, \quad \mathbf{L}=\max _{i, j=1, \ldots, N}\left|L_{j}^{i}\right|, \\
\mathbf{U}_{\mathbf{0}} & =\max _{i=1, \ldots, N}\left|U_{0}^{i}\right|
\end{array}
$$

the respective norms of $\mathbb{Q}, L$ and $U_{0}$.

The 0 -th order of [33] gives:

$$
\begin{aligned}
-U_{1}^{i} & =\sum_{j=1}^{N} \sum_{l=1}^{N} \mathbb{Q}_{j l}^{i} U_{0}^{j} U_{0}^{l}+\sum_{j=1}^{N} L_{j}^{i} a_{0}^{j}, \\
\left|U_{1}^{i}\right| & <\mathbf{Q} \sum_{j=1}^{N} \sum_{l=1}^{N} \mathbf{U}_{\mathbf{0}}{ }^{2}+\mathbf{L} \sum_{j=1}^{N} \mathbf{U}_{\mathbf{0}} \\
\left|U_{1}^{i}\right| & <\mathbf{Q} N^{2} \mathbf{U}_{\mathbf{0}}{ }^{2}+\mathbf{L} N \mathbf{U}_{\mathbf{0}} .
\end{aligned}
$$

If

$$
E=\left(\mathbf{Q} N^{2} \alpha^{2}+\mathbf{L} N \alpha\right)
$$

where $\alpha=\max \left(\mathbf{U}_{\mathbf{0}}, 1\right)$ then

$$
\left|U_{1}^{i}\right|<E, \quad i=1, \ldots, N .
$$


We will prove by recurrence that $\left|U_{k}^{i}\right|<E^{k}$ for all $i=1, \ldots, N$ and $k \in \mathbb{N}$.

The property holds when $k=1$. It is assumed true for all $i=1, \ldots, N$ and $k \leq k_{0}$, with $k_{0} \in \mathbb{N}$. Hence, at the order $k_{0}$ of [33], one has:

$$
\begin{aligned}
\left(k_{0}+1\right)\left|U_{k_{0}+1}^{i}\right| & =\left|\sum_{j=1}^{N} \sum_{l=1}^{N} \mathbb{Q}_{j l}^{i}\left(\sum_{r=0}^{k_{0}} U_{r}^{j} U_{k_{0}-r}^{l}\right)+\sum_{j=1}^{N} L_{j}^{i} U_{k_{0}}^{j}\right| \\
\left(k_{0}+1\right)\left|U_{k_{0}+1}^{i}\right| & <\mathbf{Q} \sum_{j=1}^{N} \sum_{l=1}^{N} \sum_{r=0}^{k_{0}} E^{r} E^{k_{0}-r}+\mathbf{L} \sum_{j=1}^{N} E^{k_{0}} \\
& <\mathbf{Q} N^{2}\left(k_{0}+1\right) E^{k_{0}}+\mathbf{L} N E^{k_{0}} .
\end{aligned}
$$

Hence,

$$
\left|U_{k_{0}+1}^{i}\right|<\left(\mathbf{Q} N^{2}+\mathbf{L} N \frac{1}{l+1}\right) E^{k_{0}}
$$

and then, for all $i=1, \ldots, N$,

$$
\left|U_{k_{0}+1}^{i}\right|<E^{k_{0}+1} \text {. }
$$

Consequently, the property is also true for $k=k_{0}+1$. This proves that

$$
\left|U_{k}^{i}\right|<E^{k}, \quad \forall i=1, \ldots, N, k \in \mathbb{N} .
$$

In summary, the quantity $t_{c}=1 / E$ where $E$ is defined by relation [41] is an estimation of the convergence radius of the series [32]. It decreases with $1 / N^{2}$ (or in $1 / N$ ). This convergence radius may then be very small for a high dimensional problem. For an infinite-dimensional (i.e. continuous) problem, the convergence radius may vanish (heat equation). 